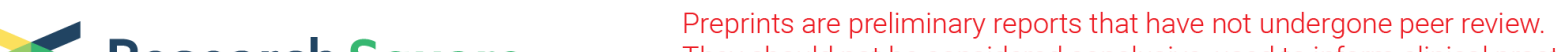 Research Square
They should not be ons sidered conllusive, used to inform clinical practice,
or referenced by the media as validated information.
}

\section{Prognostic Value of Genes Related with Tumor Microenvironment in Ovarian Cancer}

\section{Shimei Li}

Sun Yat-Sen Memorial Hosptial, Sun Yat-Sen University

Jiyi Yao

Sun Yat-Sen Memorial Hospital, Sun Yat-Sen University

Shen Zhang

Sun Yat-Sen Memorial Hospital, Sun Yat-Sen University

Xinchuan Zhou

Sun Yat-Sen Memorial Hospital, Sun Yat-Sen University

\section{Xinbao Zhao}

Sun Yat-Sen Memorial Hospital, Sun Yat-Sen University

$\mathrm{Na} \mathrm{Di}$

Sun Yat-Sen Memorial Hospital, Sun Yat-Sen University

Shaoyun Hao ( $\nabla$ haoshaoyun82@163.com )

Sun Yat-Sen Memorial Hospital, Sun Yat-Sen University

Hui Zhi ( $D$ zhihui181@126.com)

Sun Yat-sen Memorial Hospital, Sun Yat-sen University https://orcid.org/0000-0001-9708-7998

\section{Research article}

Keywords: Ovarian cancer, tumor microenvironment, stromal scores, prognosis, TCGA

Posted Date: May 29th, 2020

DOI: https://doi.org/10.21203/rs.3.rs-31574/v1

License: (c) (1) This work is licensed under a Creative Commons Attribution 4.0 International License.

Read Full License 


\section{Abstract}

Background Ovarian cancer (OV) is the fifth leading cause of cancer death among females. Growing evidence supports a key role of tumor microenvironment in growth, progress, and metastasis of OV. However, the impacts of gene expression signatures related with OV microenvironment on prognosis have not been well-established. This study aimed to apply ESTIMATE algorithm to extract genes related with tumor microenvironment that predicted poor outcomes in OV patients. Methods The gene expression profile of OV samples were downloaded from The Cancer Genome Atlas (TCGA) database. The immune scores and stromal scores of $469 \mathrm{OV}$ samples were available based on the ESTIMATE algorithm. To better understand impacts of gene expression signatures related with OV microenvironment on prognosis, these samples were categorized based on their ESTIMATE scores into high and low score groups. A different OV cohort from the Gene Expression Omnibus (GEO) database was used for external validation. Results The molecular subtypes in OV patients were correlated with stromal scores, in which the mesenchymal subtype had the highest stromal scores $(p<0.0001)$. Poor prognosis were found in patients (especially for patients with overall survivals $(O S)<5$ years) with higher stromal score $(p=$ 0.0376). 449 differentially expressed genes (DEGs) in stromal scores group were identified and 26 DEGs were significantly associated with poor prognosis in OV patients $(p<0.05)$. Eventually, 6 genes have further validated to be significantly associated with poor outcomes in 40 patients from a different $\mathrm{OV}$ cohort of GEO database $(p<0.05)$. Conclusion In this study, several genes related with tumor microenvironment that predicted poor prognosis in OV patients were extracted. In addition, some previously overlooked genes could be potential prognostic biomarkers for OV.

\section{Background}

Ovarian cancer (OV) is a leading cause of cancer death among females [1]. It is characterized by a high degree of heterogeneity and a high risk of relapse, especially for high-grade serous ovarian carcinoma (HGS-OvCa) [2]. In 2018, there was about 22,240 new cases diagnosed and 14,070 deaths from the OV in the United States [3].

As the most lethal gynecological malignancy, OV tissue includes not only tumor cells but also non-tumor cells in the microenvironment. Tumor microenvironment represents a cellular environment where the tumor exists, consisting of immune cells, stromal cells, extracellular matrix component (EMC) and exosomes [4]. Among them, immune and stromal cells are major components. Growing evidence supports a key role of tumor microenvironment in growth, progress, and metastasis of OV, hence the prognosis [5]. However, the impacts of gene expression signatures related with OV microenvironment on prognosis remain unknown. Based on gene expression profiles, The Cancer Genome Atlas (TCGA) network classified OV into four molecular subtypes: differentiated, immunoreactive, mesenchymal and proliferative in TCGA database, which were associated with patients survival [6]. BRCA1 has an important role in DNA damage repair, chromatin remodeling, cell cycle control, and transcriptional regulation and BRCA2 mainly acts on homologous recombination[7]. Although there have been many reports about the 
relationship between BRCA1/BRCA2 mutations and OV prognosis, the prognostic significance of BRCA1 mutations have not been well illuminated.

ESTIMATE (Estimation of STromal and Immune cells in MAlignant Tumor tissues using Expression data) has been proposed to be a new algorithm to score the fraction of immune and stromal cells in tumor tissues, then predict the infiltration level of immune and stromal cells and tumor purity based on gene expression signatures from the TCGA database [8]. Despite several reports have used the ESTIMATE algorithm in some tumors, such as breast cancer [9], head and neck squamous cell carcinoma [10], Glioblastoma multiforme [11], and gastric cancer [12], its application in OV has not been studied in detail.

In this study, we applied ESTIMATE algorithm to mine genes related with tumor microenvironment that predicted poor outcomes in OV patients. Notablely, we have further validated these genes by a different OV cohort from the Gene Expression Omnibus (GEO) database (Series GSE32063).

\section{Materials And Methods}

\section{Data Preparation}

Level 3 date of gene expression profile of OV samples were downloaded from the TCGA data coordination center (https://tcga-data.nci.nih.gov/tcga/), using the Affymetrix HT Human Genome U133a (HT-HG-U133A) microarray platform on September 8, 2017. Clinical data of OV samples, including gender, age, histological type, clinical stage, neoplasm histologic grade, molecular subtypes, mutation status, and survival information were also obtained from TCGA database. ESTIMATE scores, including immune scores and stromal scores of 469 OV samples were available based on the ESTIMATE algorithm to the normalized expression matrix. A dataset from the GEO database were used for validation in this study, including 40 OV patients from Series GSE32063.

\section{Identification and analysis of differentially expressed genes (DEGs)}

According to the ESTIMATE scores obtained from the ESTIMATE algorithm, all OV samples were divided into two groups, namely high (immune or stromal) scores group and low (immune or stromal) scores group. R package limma was used for the identification of DEGs [13]. The DEGs between two groups (high group vs. low group) were screened based on the following cut-offs: false discovery rate (FDR) adjusted $p$-value $<0.05$ and fold change $(F C)>1.5$. "Upregulated DEGs" represented the genes that were upregulated in the high group compared with the low group and "downregulated DEGs" showed the genes that were downregulated in the high group compared with the low group.

\section{Heatmaps and clustering analysis}

ClustVis, as a web tool, was used to produce heatmaps and principal component analysis (PCA) plots of DEGs distribution between high and low ESTIMATE scores groups [14].

\section{Construction of volcano plots}


Volcano plots were used for determining the distribution of upregulated DEGs and downregulated DEGs.

\section{Construction of Venn diagrams}

The upregulated DEGs and downregulated DEGs shared in two groups (immune scores group vs. stromal scores group) were identified by Venn diagrams (Venn 2.1.0), respectively.

\section{Gene ontology (GO) function and Kyoto Encyclopedia of Genes and Genomes (KEGG) pathway enrichment analysis of DEGs}

GO function (biological processes (BP), molecular functions (MF), and cellular components (CC)) and KEGG pathway enrichment analysis of DEGs were assessed by the Database for Annotation, Visualization and Integrated Discovery (DAVID) [15]. An FDR adjusted $p$-value $<0.05$ was considered to be statistically significant.

\section{Construction of protein-protein interaction (PPI) network}

The PPI network of upregulated DEGs in the stromal group was constructed by the STRING database [16] and Cytoscape software 3.6.1 [17].

\section{Overall survival (OS) curve}

The relationship between gene expression levels of DEGs and OS in OV patients was analyzed by KaplanMeier survival curve with a log-rank (Mantel-Cox) test.

\section{Statistical Analyses}

All statistical analysis were carried out using GraphPad Prism 5 (Version 5.01) and R version 3.5.1. The data analyses in this study were performed by standard statistical tests whenever appropriate. Comparisons between groups were evaluated by unpaired $t$ test and one-way analysis of variance. $\mathrm{P}<$ 0.05 was deemed to be statistical significant.

\section{Results}

\section{Correlation of ESTIMATE scores with OV prognosis}

The gene expression profiles and clinical date of $469 \mathrm{OV}$ female patients diagnosed pathologically between 1992 and 2009 were downloaded from the TCGA database. Among them, 63 (13.4\%) cases were of differentiated subtype, 80 (17.1\%) cases of immunoreactive subtype, $64(13.6 \%)$ cases of mesenchymal subtype, $74(15.8 \%)$ cases of proliferative subtype, and $188(40.1 \%)$ cases of unknown molecular subtype. ESTIMATE algorithm was used to evaluate infiltration of immune and stromal cells and tumor purity. According to ESTIMATE algorithm, immune scores ranged from -1498.58 to 2774.16 , and stromal scores were distributed between -1988.05 to 1837.43 , respectively. In short, immune scores of patients were higher than stromal scores in the entire OV cohort. Immunoreactive subtype showed the 
highest average immune scores of 4 subtypes, followed by mesenchymal subtype, differentiated subtype, and proliferative subtype (Fig. 1A, p < 0.0001). Similarly, mesenchymal subtype ranked the highest average stromal scores, followed by immunoreactive subtype, differentiated subtype, and proliferative subtype (Fig. 1B, p < 0.0001), suggesting that both immune and stromal scores were obviously correlated with the classification of molecular subtypes in OV patients. The proliferative subtype patients had the lowest immune scores and stromal scores.

In this study, there were 10 BRCA1 mutant patients with OV, 287 BRCA1 wild-type patients, and 172 patients of unknown status. In addition, there were 9 BRCA2 mutant patients, 288 BRCA2 wild-type patients, and 172 patients of unknown status. We drew the distribution of ESTIMATE scores between BRCA1/2 mutant and wild-type patients with OV. However, no significant difference was found in ESTIMATE scores between BRCA1/2 mutant and wild-type patients (Fig. 1C-F).

To better understand the correlation of ESTIMATE scores with OV prognosis, we categorized the 469 OV patients into high and low scores groups according to the top half of 235 samples with higher ESTIMATE scores and the bottom half of 234 samples with lower ESTIMATE scores. We found that patients with low immune scores showed no difference in median survival compared with patients with high scores $(p=$ 0.5707 , Fig. $1 \mathrm{H}$ ). Median survival of patients with high stromal scores was lower than the patients with low scores based on Kaplan-Meier curve, although it showed no statistical significance $(p=0.1145)$. And the trend was mainly reflected in the shorter median survival (OS $<5$ years) $(p=0.0376$, Fig. 1G).

\section{Comparison of DEGs with ESTIMATE scores}

To explore the association of gene expression signatures with ESTIMATE scores, we compared gene expression profiles of 469 OV samples downloaded from TCGA database. Heatmaps and PCA plots demonstrated visualized distribution of gene expression profiles between high and low ESTIMATE scores groups in Fig. 2A-D. A total of 487 genes were identified to be DEGs in immune scores group. Among them, there were 442 upregulated DEGs and 45 downregulated DEGs in the high group compared with the low group of immune scores based on $p<0.05$ and $F C>1.5$. On the other hand, 449 genes were identified to be DEGs in stromal scores group. Of these, there were 428 upregulated DEGs and 21 downregulated DEGs in the high group compared with the low group of stromal scores based on $p<0.05$ and $F C>1.5$. Similarly, volcano plots (Fig. 2E, F) showed the distribution of upregulated DEGs and downregulated DEGs in immune scores and stromal scores groups. Furthermore, Venn diagrams displayed 287 upregulated DEGs shared in the immune scores and stromal scores groups, and 13 downregulated DEGs shared (Fig. 2G, H). Since only stromal scores showed distinct association with OV prognosis, we determined to mainly explore the DEGs in stromal scores group for the subsequent analysis in this study.

To predict the potential function of 428 upregulated DEGs (Table 1) in stromal scores group, we performed the GO function and KEGG pathway enrichment analysis of these genes. There were $355 \mathrm{GO}$ terms of $\mathrm{BP}, 64 \mathrm{GO}$ terms of $\mathrm{CC}$, and $76 \mathrm{GO}$ terms of MF indicated significant difference based on FDR < 0.05 and $\log (\mathrm{FDR})>1.301$. Top $10 \mathrm{GO}$ terms were identified, such as immune response, extracellular space, and extracellular matrix (ECM) structural constituent (Fig. 3A-C). In addition, a total of 54 KEGG 
pathway categories showed to be significant based on FDR $<0.05$ and $\log (F D R)>1.301$. Top 10 KEGG pathway categories were identified, such as staphylococcus aureus infection, phagosome, ECM-receptor interaction, which were related with immune response (Fig. 3D).

\section{Correlation of expression of DEGs with OV prognosis}

To estimate the possible role of DEGs in OS, we ploted Kaplan-Meier survival curves with log-rank (Mantel-Cox) test. Among the 428 upregulated DEGs in the stromal scores group, a total of 44 DEGs were screen out to be significantly associated with OV prognosis. Of these 44 DEGs, 26 DEGs (hazard ratio $(H R)>1$, Table 2$)$ obviously predicted poor outcomes for $\mathrm{OV}(\mathrm{p}<0.05$, selected genes are shown in Fig. $4 A-F)$.

\section{PPI network of prognostic DEGs}

To functionally explore the interactions between these prognostic DEGs, we constructed PPI network by the STRING database and Cytoscape software, which consisted of 41 nodes. We defined the color of node continuously by log (FC) value of prognostic DEGs in the network. Similarly, we identified the size of node continuously based on degree value which was the number of connections the node and other nodes. In this PPI network, MMP9, CXCL10, GZMB, CXCL9, CXCL11, CXCL13, C5AR1, GBP1, CD2, GBP2 and CX3CR1 were the notable nodes with higher degree values, because they showed strong association with other nodes (Fig. 5).

\section{Validation of prognostic DEGs in a external cohort}

To determine whether the prognostic DEGs identified from the TCGA database also have significant prognostic value in a different OV cohort, we obtained and analyzed gene expression signatures of a dataset with 40 OV samples from the GEO database (Series GSE32063). Eventually, 6 genes, including $\mathrm{CH} 25 \mathrm{H}, \mathrm{CX} 3 \mathrm{CR} 1, \mathrm{GFPT} 2, \mathrm{NBL} 1, \mathrm{TFPI}$, and ZFP36 were verified to be significantly correlated with poor outcomes $(p<0.05$, Fig. $6 A-F)$.

\section{Discussion}

Because the early symptoms and signs of OV are atypical or non-existent, most patients are initially diagnosed at an advanced stage with low five-year survival rates [18]. Therefore, it is necessary to develop new methods to screen for early OV and new treatment strategies. With the impact of tumor microenvironment on OV prognosis has been increasingly emphasized [19], the interaction between tumor microenvironment components and OV prognosis was urgently needed to better understand. In this study, we identified several genes related with tumor microenvironment that predicted poor outcomes in OV patients from the TCGA database.

The mesenchymal subtype is featured by the overexpression of several genes related with activated stroma at the molecular level [20], although the stromal cells of the mesenchymal subtype have not been well described. It has been gradually recognized that stromal components are not only a scaffold for 
integrity of tissue structure, but also play a role in tumor genesis, growth, invasion and metastasis $[21,22]$. In this study, the molecular subtypes in OV patients were found to be significantly correlated with the level of stromal scores, in which the mesenchymal subtype had the highest stromal scores. We further compared median survival of high and low stromal scores groups, and found that patients with high stromal scores were shown to be associated with low median survival, indicating poor prognosis. Therefore, our results demonstrated mesenchymal subtype was associated with poor outcomes, which was consistent with previous reports [23]. Stromal components in tumor microenvironment may significantly contribute to the behaviors of OV with mesenchymal subtype.

The prognostic significance of BRCA1/2 mutations in OV is still unclear. Previous report found a better prognosis for BRCA2 mutant patients, with no significant difference in prognosis for BRCA1 mutant patients compared with wild-type patients [24]. But several studies have showed that patients with BRCA1 and BRCA2 mutations had a better outcome compared with wild-type patients [25,26], whereas other studies have demonstrated no significant difference [27]. Our study also showed no significant prognostic difference, but small sample sizes of BRCA1/2 mutations might lead to inaccurate estimates of survival.

Increasing evidences have showed that tumor development is influenced not only by its internal tumor cells but also by its external tumor microenvironment components [28-31]. However, these studies have focused limited attention on stromal components in the tumor microenvironment. In this study, through gene expression profile of 469 OV samples, we firstly analyzed 449 DEGs identified from the comparison between high and low stromal scores groups, some of which participated in tumor microenvironment, as shown by GO function and KEGG pathway enrichment analysis. This was consistent with previous studies that stromal components were correlated with the building of tumor microenvironment in OV [3234].

Then, we performed survival analysis of these 449 genes and determined that 44 DEGs were significantly associated with OV prognosis, of which 26 DEGs obviously predicted poor prognosis. In addition, we constructed PPI network of 44 DEGs and found that these genes were highly interrelated. CX3CR1 [35], CRYAB [36,37], TGFBI [38], TFPI2 [39], and GFPT2 [40] have been reported to play a vatal role in stimulating tumor proliferation, invasion, and metastasis in OV patients, predicting poor prognosis. Additionally, previous studies have shown that PLK2 [41] and MGP [42] were associated with drug resistance.

Eventually, we have further validated 6 genes $(\mathrm{CH} 25 \mathrm{H}, \mathrm{CX} 3 \mathrm{CR} 1, \mathrm{NBL} 1, \mathrm{TFPI} 2, \mathrm{GFPT} 2$ and ZFP36) related with tumor microenvironment to be significantly associated with poor outcomes in 40 patients from a different OV cohort of GEO database. Of these 6 genes, as mentioned above, it has been demonstrated that the expression of CX3CR1, TFPI2 and GFPT2 were associated with poor prognosis in OV patients. Although the remaining 3 genes, including $\mathrm{CH} 25 \mathrm{H}, \mathrm{NBL} 1$, and ZFP36, have not previously showed correlation with OV prognosis, our results supported that these genes may be potential prognostic biomarkers for $\mathrm{OV}$. As a reticulum-associated membrane protein, $\mathrm{CH} 25 \mathrm{H}$ has been shown to inhibit 
infection of several viruses through catalyzing cholesterol converting into 25 -hydroxycholesterol $(25 \mathrm{HC})$ [43]. It has been previously demonstrated that the overexpression of NBL1 suppressed tumor growth [44]. Furthermore, ZFP36 has been reported to play a key role in the post-transcriptional regulation of tumor necrosis factor (TNF) and the modulation of mRNA stability [45].

\section{Conclusion}

we applied ESTIMATE algorithm based on stromal scores to extract several genes related with tumor microenvironment from TCGA database. Notablely, we have further validated these genes by a different OV cohort from the GEO database, which may be significantly associated with poor prognosis in OV patients. In addition, some previously overlooked genes could be potential prognostic biomarkers for OV. However, there were also some limitations in this study. First, fewer genes have been shown to involve in OV prognosis, possibly due to the small sample size of OV cohort for validation. Second, considering that this was a retrospective study on the basis of public databases, it was difficult to include regional differences, since the database of this study was from the United States, while the database for validation was from Japan. Therefore, well-designed, prospective, and multicenter clinical studies are still imperative to further verify the prognostic value of these genes related with tumor microenvironment by large data-based analyses, which may help us to develop novel prognostic biomarkers and therapeutic targets for OV in clinical practice.

\section{Abbreviation}

OV: ovarian cancer; TCGA: Cancer Genome Atlas; GEO: Gene Expression Omnibus; OS: overall survivals; DEGs: differentially expressed genes; HGS-OvCa: high-grade serous ovarian carcinoma; EMC: extracellular matrix component; FDR: false discovery rate; PCA: principal component analysis; GO: Gene ontology; KEGG: Kyoto Encyclopedia of Genes and Genomes; BP: biological processes; MF: molecular functions; CC: cellular components; DAVID: Database for Annotation, Visualization and Integrated Discovery; PPI: protein-protein interaction; HR: hazard ratio; $25 \mathrm{HC}$ : 25-hydroxycholesterol; TNF: tumor necrosis factor.

\section{Declarations}

\section{Acknowledgements}

Not applicable.

\section{Author contributions}

Conception and design: Hui Zhi, Shimei Li, Shaoyun Hao

Development of methodology: Hui Zhi, Shimei Li, Shaoyun Hao 
Acquisition of data: Hui Zhi, Shimei Li, Shaoyun Hao, Jiyi Yao, Shen Zhang, Xinchuan Zhou, Xinbao Zhao, $\mathrm{Na} \mathrm{Di}$

Analysis and interpretation of data: Hui Zhi, Shimei Li, Shaoyun Hao, Jiyi Yao, Shen Zhang, Xinchuan Zhou, Xinbao Zhao, Na Di

Writing, review, and/or revision of the manuscript: Shimei Li, Hui Zhi

Study supervision: Hui Zhi

All authors read and approved the final manuscript.

\section{Funding}

This work was supported by grant 201804010237 from the Science and Technology Program of Guangzhou, China.

\section{Availability of data and materials}

The datasets generated during the current study are available in the TCGA repository (https://tcgadata.nci.nih.gov/tcga/). TCGA are publicly available databases.

\section{Ethics approval and consent to participate}

All procedures performed in studies involving human participants were in accordance with the ethical standards of the institutional and/or national research committee and with the 1964 Helsinki declaration and its later amendments or comparable ethical standards. The study has been approved by the ethics committee of Sun Yat-Sen Memorial Hospital of Sun Yat-Sen University.

\section{Consent for publication}

Not applicable.

\section{Competing interests}

The authors declare no conflicts of interest.

\section{References}

1. González-Martín Antonio, Pothuri Bhavana, Vergote Ignace et al. Niraparib in Patients with Newly Diagnosed Advanced Ovarian Cancer. N. Engl. J. Med., 2019; 381: 2391-402.

2. Zhang Allen W, McPherson Andrew, Milne Katy et al. Interfaces of Malignant and Immunologic Clonal Dynamics in Ovarian Cancer. Cell, 2018; 173: 1755-69.e22. 
3. Torre Lindsey A, Trabert Britton, DeSantis Carol E et al. Ovarian cancer statistics, 2018. CA Cancer J Clin, 2018; 68: 284-96.

4. Luo Zhongyue, Wang Qiu, Lau Wayne Bond et al. Tumor microenvironment: The culprit for ovarian cancer metastasis? Cancer Lett., 2016; 377: 174-82.

5. Pearce Oliver M T, Delaine-Smith Robin M, Maniati Eleni et al. Deconstruction of a Metastatic Tumor Microenvironment Reveals a Common Matrix Response in Human Cancers. Cancer Discov, 2018; 8: 304-19.

6. Leong Huei San, Galletta Laura, Etemadmoghadam Dariush et al. Efficient molecular subtype classification of high-grade serous ovarian cancer. J. Pathol., 2015; 236: $72-7$.

7. Bolton Kelly L, Chenevix-Trench Georgia, Goh Cindy et al. Association between BRCA1 and BRCA2 mutations and survival in women with invasive epithelial ovarian cancer. JAMA, 2012; 307: 382-90.

8. Yoshihara Kosuke, Shahmoradgoli Maria, Martínez Emmanuel et al. Inferring tumour purity and stromal and immune cell admixture from expression data. Nat Commun, 2013; 4: 2612.

9. Chen Ching-Hsuan, Lu Yen-Shen, Cheng Ann-Lii et al. Disparity in Tumor Immune Microenvironment of Breast Cancer and Prognostic Impact: Asian Versus Western Populations. Oncologist, 2020; 25: e16-e23.

10. Lin Ching Ying, Kwon Hyunwoo, Rangel Rivera Guillermo 0 et al. Sex Differences in Using Systemic Inflammatory Markers to Prognosticate Patients with Head and Neck Squamous Cell Carcinoma. Cancer Epidemiol. Biomarkers Prev., 2018; 27: 1176-85.

11. Jia Di, Li Shenglan, Li Dali et al. Mining TCGA database for genes of prognostic value in glioblastoma microenvironment. Aging (Albany NY), 2018; 10: 592-605.

12. Wang Hao, Wu Xiaosheng, Chen Yiming, Stromal-Immune Score-Based Gene Signature: A Prognosis Stratification Tool in Gastric Cancer. Front Oncol, 2019; 9: 1212.

13. Ritchie Matthew E, Phipson Belinda, Wu Di et al. limma powers differential expression analyses for RNA-sequencing and microarray studies. Nucleic Acids Res., 2015; 43: e47.

14. Metsalu Tauno, Vilo Jaak, ClustVis: a web tool for visualizing clustering of multivariate data using Principal Component Analysis and heatmap. Nucleic Acids Res., 2015; 43: W566-70.

15. Huang Da Wei, Sherman Brad T, Lempicki Richard A, Systematic and integrative analysis of large gene lists using DAVID bioinformatics resources. Nat Protoc, 2009; 4: 44-57.

16. Szklarczyk Damian, Franceschini Andrea, Wyder Stefan et al. STRING v10: protein-protein interaction networks, integrated over the tree of life. Nucleic Acids Res., 2015; 43: D447-52.

17. Shannon Paul, Markiel Andrew, Ozier Owen et al. Cytoscape: a software environment for integrated models of biomolecular interaction networks. Genome Res., 2003; 13: 2498-504.

18. Lu Mingxia, Henry Claire E, Lai Haiwang et al. A new 3D organotypic model of ovarian cancer to help evaluate the antimetastatic activity of RAPTA-C conjugated micelles. Biomater Sci, 2019; 7: 1652-60.

19. Coukos G, Tanyi J, Kandalaft L E, Opportunities in immunotherapy of ovarian cancer. Ann. Oncol., 2016; null: i11-i15. 
20. Zhang Qing, Wang Chen, Cliby William A, Cancer-associated stroma significantly contributes to the mesenchymal subtype signature of serous ovarian cancer. Gynecol. Oncol., 2019; 152: 368-74.

21. Hanahan Douglas, Weinberg Robert A, Hallmarks of cancer: the next generation. Cell, 2011; 144: 64674.

22. Straussman Ravid, Morikawa Teppei, Shee Kevin et al. Tumour micro-environment elicits innate resistance to RAF inhibitors through HGF secretion. Nature, 2012; 487: 500-4.

23. Torres Diogo, Kumar Amanika, Bakkum-Gamez Jamie $\mathrm{N}$ et al. Mesenchymal molecular subtype is an independent predictor of severe postoperative complications after primary debulking surgery for advanced ovarian cancer. Gynecol. Oncol., 2019; 152: 223-27.

24. Yang Da, Khan Sofia, Sun Yan et al. Association of BRCA1 and BRCA2 mutations with survival, chemotherapy sensitivity, and gene mutator phenotype in patients with ovarian cancer. JAMA, 2011; 306: 1557-65.

25. Chetrit Angela, Hirsh-Yechezkel Galit, Ben-David Yehuda et al. Effect of BRCA1/2 mutations on longterm survival of patients with invasive ovarian cancer: the national Israeli study of ovarian cancer. J. Clin. Oncol., 2008; 26: 20-5.

26. Cass Ilana, Baldwin Rae Lynn, Varkey Taz et al. Improved survival in women with BRCA-associated ovarian carcinoma. Cancer, 2003; 97: 2187-95.

27. Buller Richard E, Shahin Mark S, Geisler John P et al. Failure of BRCA1 dysfunction to alter ovarian cancer survival. Clin. Cancer Res., 2002; 8: 1196-202.

28. Jiménez-Sánchez Alejandro, Memon Danish, Pourpe Stephane et al. Heterogeneous Tumor-Immune Microenvironments among Differentially Growing Metastases in an Ovarian Cancer Patient. Cell, 2017; 170: 927-938.e20.

29. Drakes Maureen L, Stiff Patrick J, Regulation of Ovarian Cancer Prognosis by Immune Cells in the Tumor Microenvironment. Cancers (Basel), 2018; 10: undefined.

30. Mesnage S J L, Auguste A, Genestie C et al. Neoadjuvant chemotherapy (NACT) increases immune infiltration and programmed death-ligand 1 (PD-L1) expression in epithelial ovarian cancer (EOC). Ann. Oncol., 2017; 28: 651-57.

31. Liu Rong, Hu Rong, Zeng Ying et al. Tumour immune cell infiltration and survival after platinumbased chemotherapy in high-grade serous ovarian cancer subtypes: A gene expression-based computational study. EBioMedicine, 2020; 51: 102602.

32. MacGregor Heather L, Sayad Azin, Elia Andrew et al. High expression of B7-H3 on stromal cells defines tumor and stromal compartments in epithelial ovarian cancer and is associated with limited immune activation. J Immunother Cancer, 2019; 7: 357.

33. Thuwajit Chanitra, Ferraresi Alessandra, Titone Rossella et al. The metabolic cross-talk between epithelial cancer cells and stromal fibroblasts in ovarian cancer progression: Autophagy plays a role. Med Res Rev, 2018; 38: 1235-54.

34. Kuhnert Frank, Chen Guoying, Coetzee Sandra et al. DII4 Blockade in Stromal Cells Mediates Antitumor Effects in Preclinical Models of Ovarian Cancer. Cancer Res., 2015; 75: 4086-96. 
35. Singh Santosh Kumar, Mishra Manoj Kumar, Singh Rajesh, Hypoxia-inducible factor-1a induces CX3CR1 expression and promotes the epithelial to mesenchymal transition (EMT) in ovarian cancer cells. J Ovarian Res, 2019; 12: 42.

36. Volkmann J, Reuning U, Rudelius M. et al. High expression of crystallin alpha B represents an independent molecular marker for unfavourable ovarian cancer patient outcome and impairs TRAILand cisplatin-induced apoptosis in human ovarian cancer cells. Int J Cancer, 2013; 132 (12) :282032.

37. Tan Lin, Sha Ling, Hou Ning et al. High a B-crystallin and p53 co-expression is associated with poor prognosis in ovarian cancer. Biosci. Rep., 2019; 39: undefined.

38. Karlan Beth Y, Dering Judy, Walsh Christine et al. POSTN/TGFBI-associated stromal signature predicts poor prognosis in serous epithelial ovarian cancer. Gynecol. Oncol., 2014; 132: 334-42.

39. Arakawa Noriaki, Miyagi Etsuko, Nomura Ayako et al. Secretome-based identification of TFPI2, a novel serum biomarker for detection of ovarian clear cell adenocarcinoma. J. Proteome Res., 2013; 12: 4340-50.

40. Zhou Lin, Luo Mu, Cheng Li-Juan et al. Glutamine-fructose-6-phosphate transaminase 2 (GFPT2) promotes the EMT of serous ovarian cancer by activating the hexosamine biosynthetic pathway to increase the nuclear location of $\beta$-catenin. Pathol. Res. Pract., 2019; 215: 152681.

41. Syed Nelofer, Coley Helen M, Sehouli Jalid et al. Polo-like kinase Plk2 is an epigenetic determinant of chemosensitivity and clinical outcomes in ovarian cancer. Cancer Res., 2011; 71: 3317-27.

42. Sterzyńska Karolina, Klejewski Andrzej, Wojtowicz Karolina et al. The Role of Matrix Gla Protein (MGP) Expression in Paclitaxel and Topotecan Resistant Ovarian Cancer Cell Lines. Int J Mol Sci, 2018; 19: undefined.

43. Ke Wenting, Fang Liurong, Jing Huiyuan et al. Cholesterol 25-Hydroxylase Inhibits Porcine Reproductive and Respiratory Syndrome Virus Replication through Enzyme Activity-Dependent and Independent Mechanisms. J. Virol., 2017; 91: undefined.

44. Cui Chuanjue, Zhang Hongliang, Guo Lin-Na et al. Inhibitory effect of NBL1 on PDGF-BB-induced human PASMC proliferation through blockade of PDGF $\beta$-p38MAPK pathway. Biosci. Rep., 2016; 36: undefined.

45. Mukherjee Neelanjan, Jacobs Nicholas $C$, Hafner Markus et al. Global target mRNA specification and regulation by the RNA-binding protein ZFP36. Genome Biol., 2014; 15: R12.

\section{Tables}

Table 1428 Upregulated DEGs extracted from comparison between high and low stromal scores groups 
Gene symbol

1 THBS2

2 SERPINF1

3 VCAN

$4 \quad$ FN1

5 CTSK

6 DCN

7 COL5A2

$8 \quad$ FBN1

9 COL3A1

10 COL1A1

11 FAP

12 VCAM1

13 LUM

14 AEBP1

15 EVI2A

16 MMP2

17 COL5A1

18 RAB31

19 ACTA2

20 GLIPR1 
21 COL6A3

22 COL10A1

23 POSTN

24 SPARC

25 TGFBI

26 CDH11

27 SNAI2

28 ECM1

29 COL1A2

30 PLAU

31 SFRP4

32 CRISPLD2

33 INHBA

34 OLFML2B

35 COPZ2

36 THBS1

37 LY96

38 COL11A1

39 FILIP1L

40 WIPF 1

41 PMP22 
42 COLEC12

43 CXCL12

44 CLEC2B

45 COL6A2

46 FCGR2A

47 PTPRC

48 DAB2

49 OLFML1

50 C3AR1

51 ITGA5

52 TNFAIP6

53 IL7R

54 CD163

55 GIMAP4

56 LRRC15

57 GMFG

58 EVI2B

59 HEPH

$60 \quad \mathrm{CD} 14$

61 FMO1

62 LHFP 
63 LGALS1

64 LAPTM5

65 GLT8D2

66 ECM2

67 CD53

68 GFPT2

69 VSIG4

70 EMP3

71 MS4A6A

72 FCER1G

73 SULF1

74 SAMSN1

75 PDGFRA

76 C1QB

77 ALDH1A3

78 AIF1

79 TAGLN

80 C1QA

81 F13A1

82 TDO2

83 SPHK1

Page 16/41 
84 TGFB1I1

85 PDGFRB

86 PCOLCE

$87 \quad$ EBI2

88 ASPN

89 LPPR4

90 ARHGEF6

91 LCP2

92 ITGB2

93 MMP11

94 NBL1

95 CCL11

96 MS4A4A

97 EDNRA

98 CSF2RB

99 BGN

100 TMEM158

101 FCGR1A

102 GIMAP6

103 HCK

104 SLAMF8 
105 C1S

106 SLA

107 IL10RA

108 PDLIM3

109 TYROBP

$110 \mathrm{CSF} 1 \mathrm{R}$

111 NNMT

112 GPNMB

113 ITGBL1

114 EPYC

115 PLEK

116 FYB

117 GREM1

118 SRGN

119 PSCDBP

120 CYBB

121 MNDA

122 CD93

123 TIMP3

124 FCGR2B

125 SPON2

Page 18/41 
126 COL16A1

127 ACTG2

128 SERPINE1

129 GLRX

130 HOPX

131 SRPX2

132 LY86

133 SLC31A2

134 ANGPTL2

135 LHFPL2

136 CCR1

137 OLFML3

138 PLXDC1

139 CXCL14

140 ARHGAP15

$141 \mathrm{MYO} 1 \mathrm{~F}$

142 MAFB

143 C10orf56

144 LST1

145 KLF2

146 HNT

Page 19/41 
147 RCN3

148 PAPSS2

149 C5AR1

150 CD86

151 LOX

152 LOXL1

153 DOCK2

154 GUCY1A3

155 PLAUR

156 AQP1

157 GPR65

158 CXorf9

159 EPB41L3

160 NPL

161 NT5E

162 ARHGDIB

163 LYZ

164 TMEM176B

165 RGS 1

166 NID2

167 RGS4 
168 LILRB1

169 CD48

170 NUAK1

171 FGL2

172 ISLR

173 PDPN

174 CCL 4

175 IGSF6

176 SLC12A8

177 SH2B3

178 RNASE6

179 CST7

180 HMOX1

181 DACT1

182 HTRA1

183 CD2

184 DRAM

185 ELTD1

186 CCR5

187 TLR2

188 CLEC7A

Page 21/41 
189 SMPDL3A

190 CENTA2

191 PALLD

192 HCLS1

193 SCG2

194 DPYD

195 MPP1

196 TFEC

197 CD248

198 HEG1

199 FZD1

200 CNN2

201 IGFBP4

202 TLR7

203 ACSL1

204 CD4

205 DSE

206 NCF2

207 SRPX

208 IL2RB

209 GEM

Page 22/41 
210 CALD1

211 CD3D

212 CXCL9

213 ALOX5AP

214 COL4A1

215 RAC2

216 LXN

217 SLC7A7

218 TNFSF4

219 NID1

220 BASP1

221 IRF8

222 LPXN

223 C7orf10

224 RCAN2

225 CFD

226 IGKC

227 CCL5

228 LDB2

229 APOC1

$230 \mathrm{CH} 25 \mathrm{H}$

Page 23/41 
231 OMD

232 PTRF

233 AP1S2

234 SPARCL1

235 ADAMDEC 1

236 IFI30

237 GZMA

238 MFSD1

239 GZMK

240 LTBP 2

241 MGC14376

242 FOLR2

243 STAB1

244 P2RY5

245 RGS 16

246 CCL2

247 MSN

248 F2R

249 DPYSL3

250 HLA-DPA1

251 RGS2

Page 24/41 
252 NKG7

253 CD36

254 PPAP2A

255 TMEM176A

256 COL15A1

257 C13orf15

258 IL2RG

259 MICAL2

260 CTGF

261 CD52

262 COMP

263 MMP9

264 RASSF2

265 HLA-DPB1

266 VIM

267 MOXD1

268 CTSS

269 BCL2A1

270 SEMA3C

271 CYR61

272 C1orf54

Page 25/41 
273 SPP1

274 MYLK

275 FAS

276 EFEMP 1

277 SNX10

278 TREM2

279 APOC2

280 TPM1

281 FSTL1

282 CORO1A

283 PLVAP

284 GBP2

285 TNC

286 C1QTNF3

287 IL32

288 MARCKS

289 JAM3

290 ID3

291 ICAM1

292 TUBB6

293 TRIM22

Page 26/41 
294 CD69

295 HLA-DQB1

296 KDELR3

297 AOC3

298 CTA-246H3.1

299 TWIST1

300 IGLV2-14

301 TNFAIP3

302 FABP 4

303 IGHM

304 CASP1

305 A2M

306 CILP

307 CTSO

308 ADH1B

309 MFAP5

310 GALNAC4S-6ST

311 LAMB1

312 CD44

313 SERPINA1

314 GJA1

Page 27/41 
315 EGR2

316 HLA-DRA

317 TPST2

318 MXRA8

319 CLEC5A

320 ITM2A

321 TMEPAI

322 PDGFD

323 KAL1

324 MXRA5

325 WIPI1

326 BHLHB2

327 PLS3

328 COL $4 A 2$

329 PRKD1

330 IGKV1-5

331 CD74

332 GAS1

333 CYP1B1

334 CXCL13

335 FMO2

Page 28/41 
336 LCP1

337 IGLV3-25

338 DOCK4

339 SLC2A3

340 GZMB

341 GYPC

342 RARRES1

343 HLA-DRB1

344 C11 orf75

345 SGK

346 ENPP1

347 CHN1

348 RFTN1

349 IL1B

350 HLA-B

351 MMD

352 HLA-DQA1

$353 \mathrm{CFH}$

354 SLC46A3

355 MYL9

356 RARRES2

Page 29/41 
357 SOD2

358 IGF1

359 PYCARD

360 MAN2A1

361 ST3GAL1

362 GPX3

363 CXCL10

364 LIPA

365 KLF6

366 HLA-DMA

367 FKBP11

368 MGP

369 PDGFRL

370 PLK2

371 C3

372 GBP1

373 ZFP36

374 CCL18

375 PXDN

376 FER1L3

377 THY1

Page 30/41 
378 EMP1

379 CAV1

380 KRT6A

381 NOX4

382 KLK6

383 LTB

384 AMIGO2

385 IL15

386 APOL1

387 SQRDL

388 HLA-DMB

389 UBB

390 CXCL11

391 IER3

392 TMEM45A

393 SDC1

394 MMP12

395 C2

396 CRYAB

397 TPBG

398 CXCL1

Page 31/41 
399 AHNAK2

400 UBD

401 LRRC17

$402 \mathrm{ADM}$

403 CX3CR 1

404 FAT

405 PSMB9

406 S100A8

407 FOS

408 S100A9

409 HCP5

410 PTGDS

411 TFPI2

412 SLC34A2

413 QPCT

414 ST6GALNAC5

415 ANPEP

416 CLDN16

417 ZFPM2

418 MMP7

419 MMP1

Page 32/41 
420 MT1E

421 KLK7

422 DNAJC15

423 SPON1

424 INDO

425 KRT23

426 S100A2

427 PDZK1IP1

$428 \mathrm{HP}$

Table 226 DEGs (hazard ratio $(\mathrm{HR})>1, \mathrm{p}<0.05$ ) significantly predicting poor outcomes in OV 


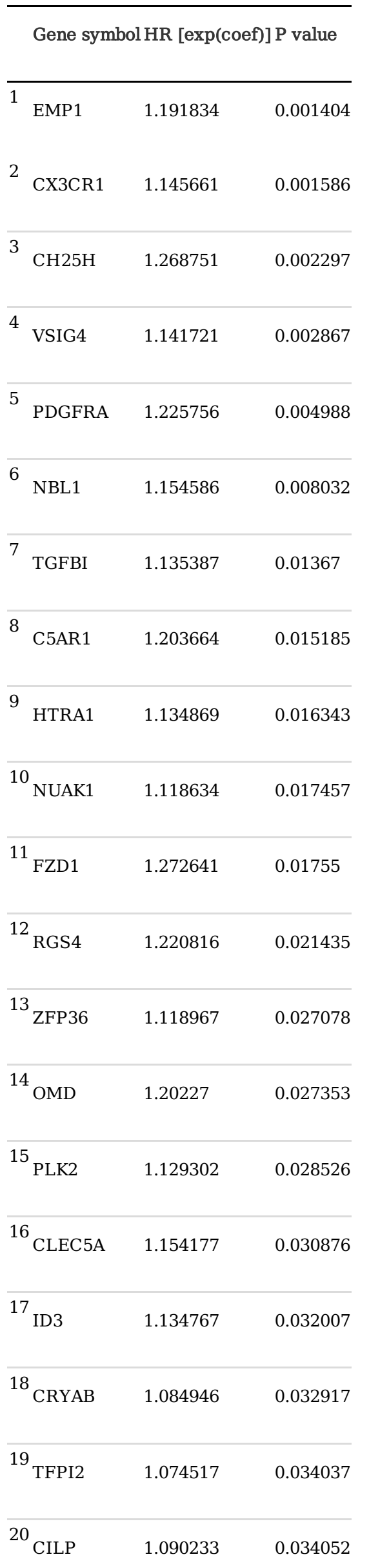

21 


\begin{tabular}{lll} 
MFAP5 & 1.075657 & 0.034413 \\
\hline 22 COL16A1 & 1.143897 & 0.040232 \\
\hline 23 PDPN & 1.140548 & 0.042185 \\
\hline 24 MGP & 1.092676 & 0.044032 \\
\hline 25 ALOX5AP & 1.076064 & 0.044146 \\
\hline 26 GFPT2 & 1.164861 & 0.045983 \\
\hline
\end{tabular}

Figures 

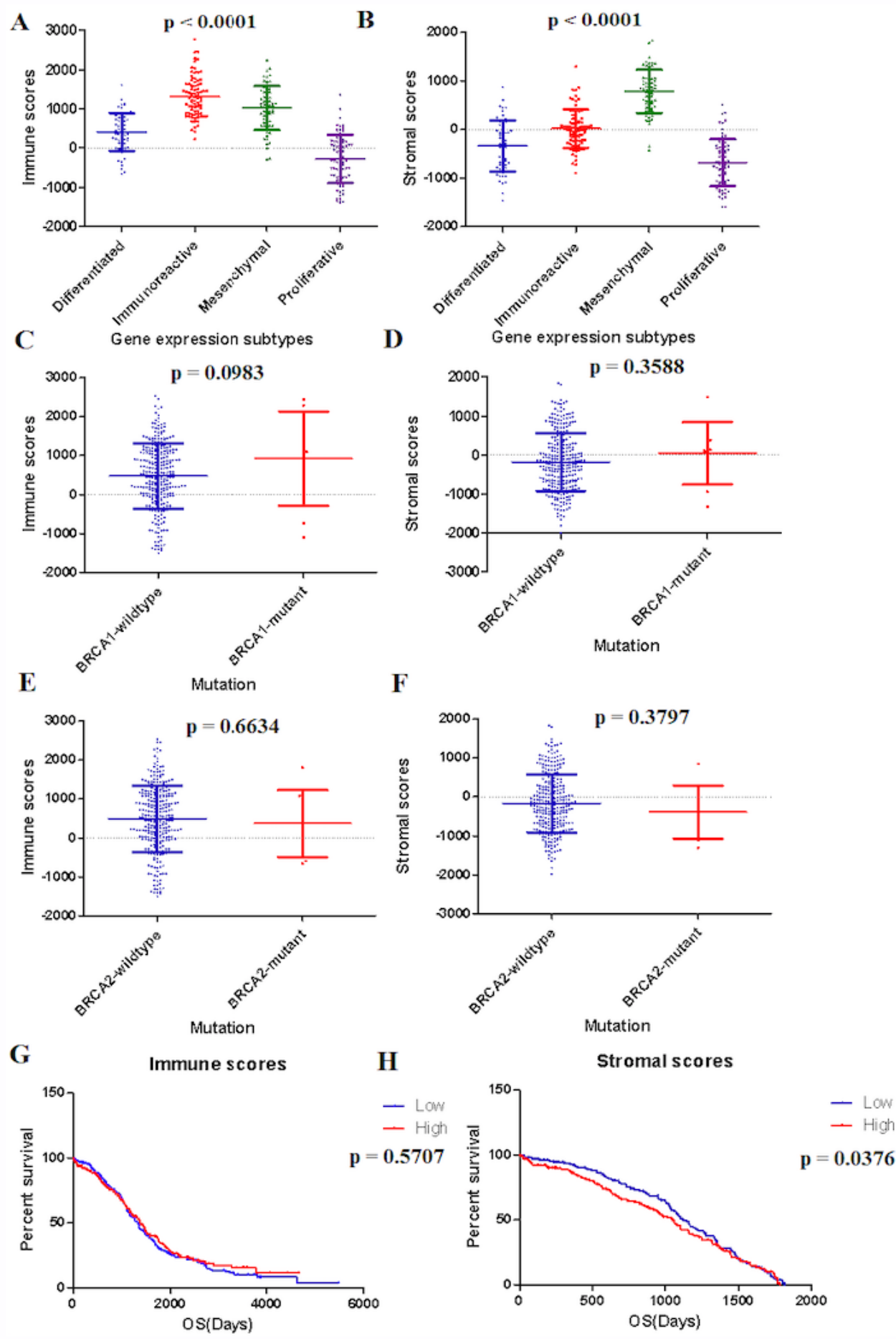

\section{Figure 1}

Correlation of ESTIMATE scores with OV prognosis. (A) Distribution of immune scores of OV subtypes ( $p$ $<0.0001)$. (B) Distribution of stromal scores of OV subtypes $(p<0.0001)$. (C) Comparisons of immune scores between BRCA1 mutant and wild-type patients $(p=0.0983)$. (D) Comparisons of strmoal scores between BRCA1 mutant and wild-type patients $(p=0.3588)$. (E) Comparisons of immune scores between BRCA2 mutant and wild-type patients $(p=0.6634)$. ( $F)$ Comparisons of strmoal scores between BRCA2 
mutant and wild-type patients ( $p=0.3797)$. (G) Kaplan-Meier survival curve for patients with low vs. high immune scores $(p=0.5707)$. $(H)$ Kaplan-Meier survival curve for patients with low vs. high stromal scores $(p=0.0376)$.
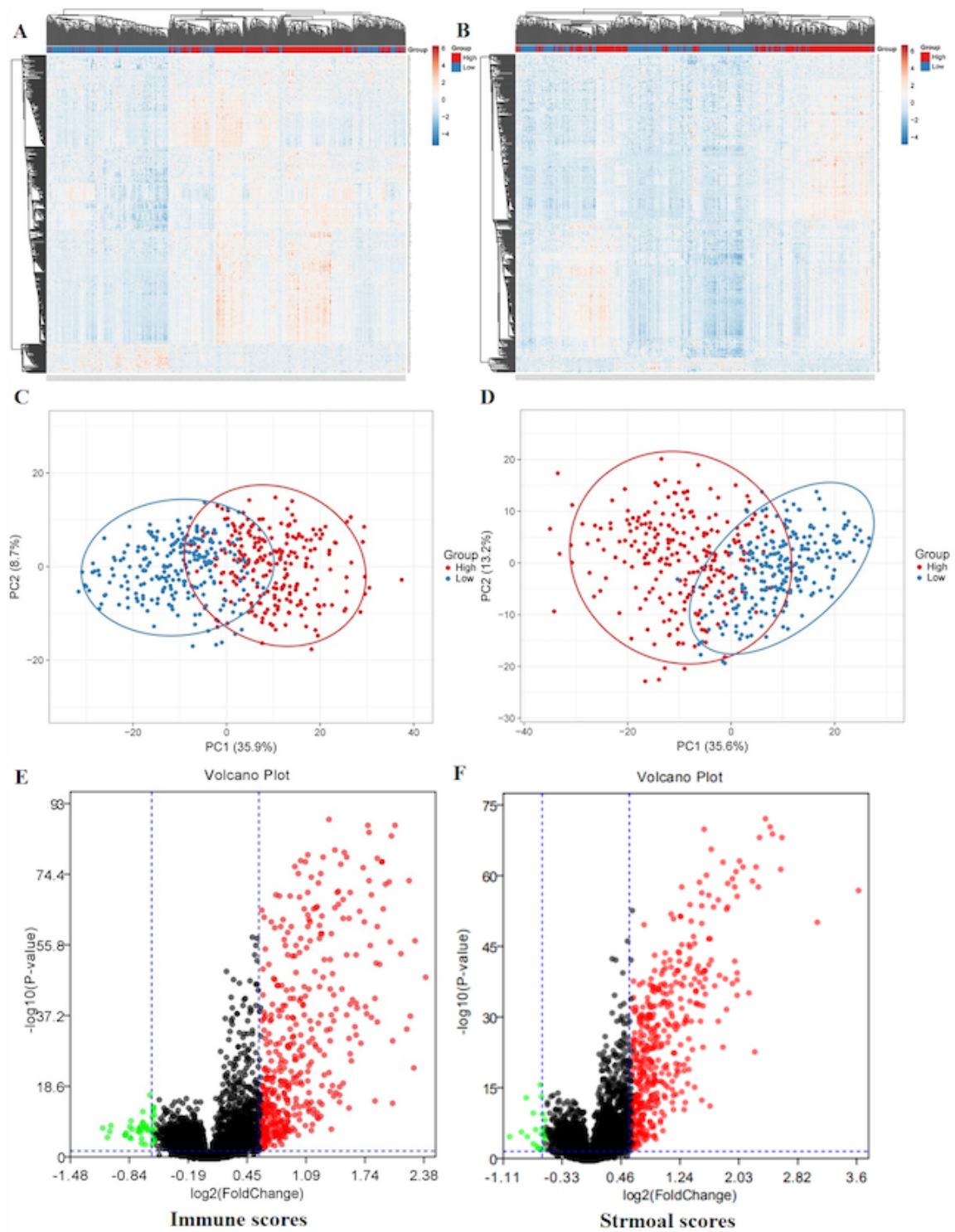

G

H
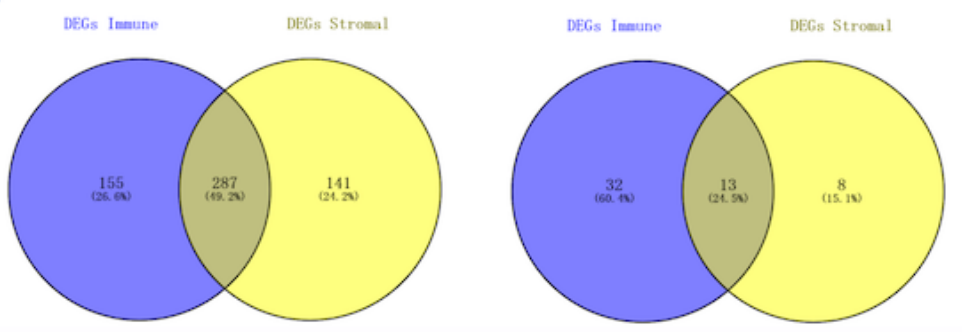

Figure 2

Comparison of DEGs with ESTIMATE scores. (A) Heatmap of the distribution of gene expression profiles between high and low immune scores groups. Genes with higher and lower expression were shown in red 
and blue, respectively, and genes at the same level of expression were showed in black. (B) Heatmap of the distribution of gene expression profiles between high and low stromal scores groups. (C) PCA plot of the distribution of gene expression profiles between high and low immune scores groups. (D) PCA plot of the distribution of gene expression profiles between high and low stromal scores groups. (E) Volcano plot of the distribution of upregulated DEGs and downregulated DEGs in immune scores group. Upregulated and downregulated DEGs were shown in red and green, respectively, the rest DEGs were shown in black. (F) Volcano plot of the distribution of upregulated DEGs and downregulated DEGs in stromal scores group. (G) Venn diagram showing the upregulated DEGs shared in the immune scores and stromal scores groups. $(H)$ Venn diagram showing the downregulated DEGs shared in two groups.

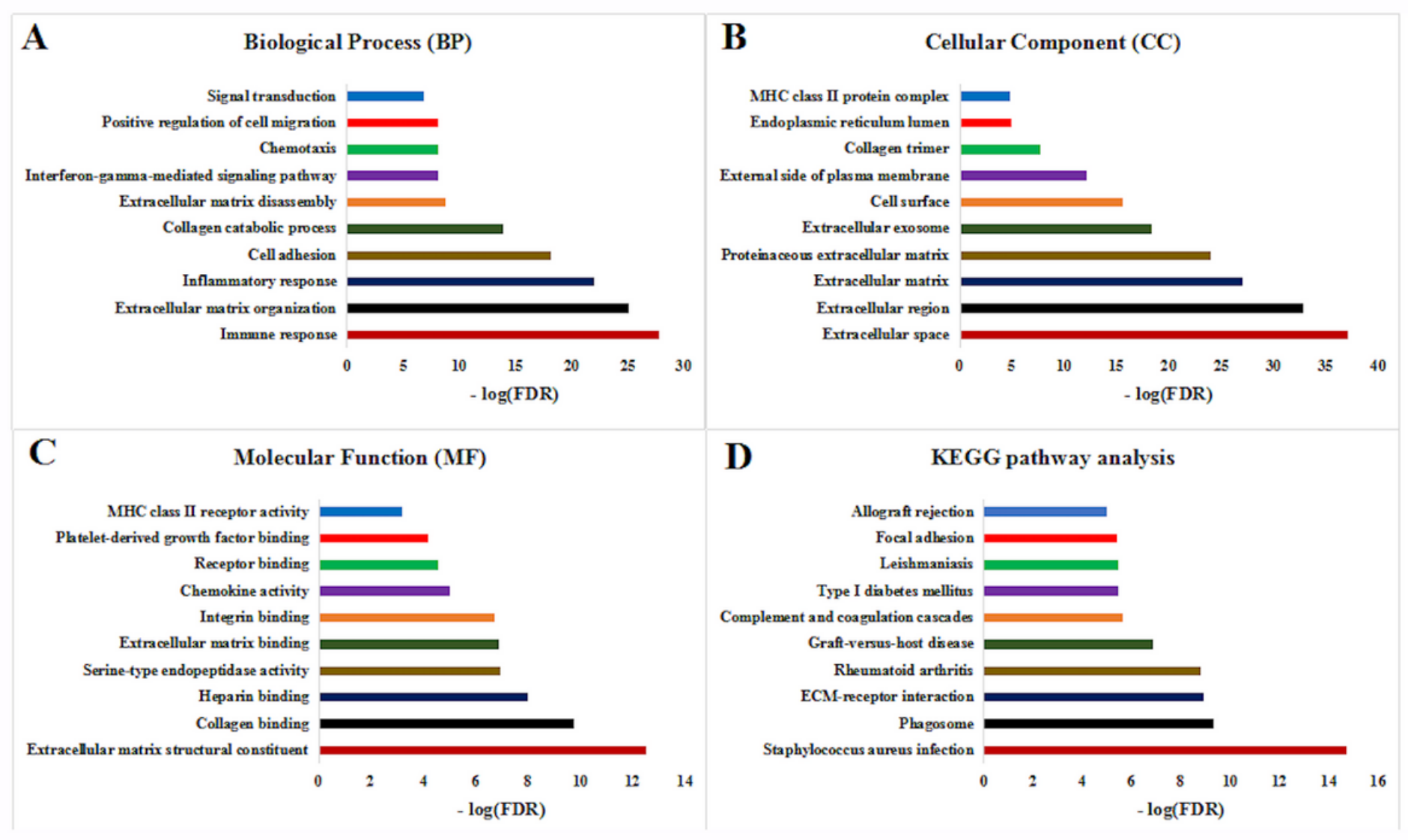

\section{Figure 3}

GO function and KEGG pathway enrichment analysis of upregulated DEGs in stromal scores group. Top $10 \mathrm{GO}$ terms of BP (A), CC (B), MF (C) and KEGG pathway categories (D). P-values were adjusted by FDR. 


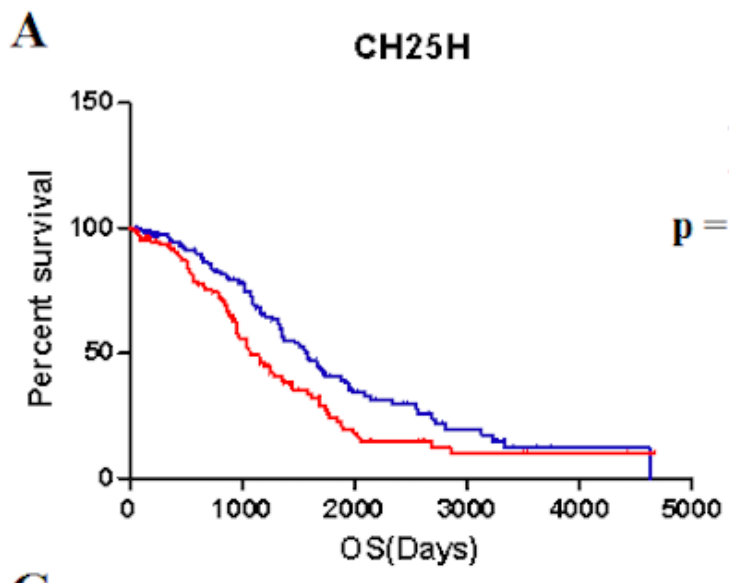

C
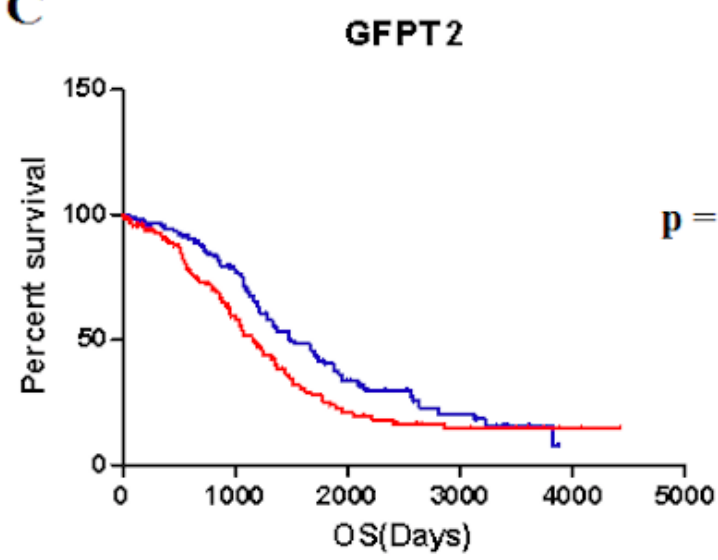

$\mathbf{E}$

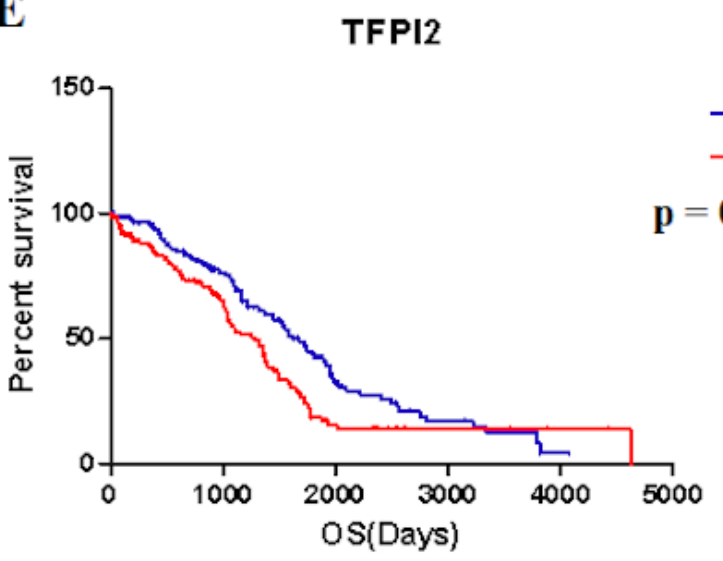

B

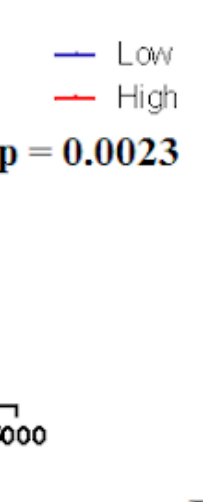

D

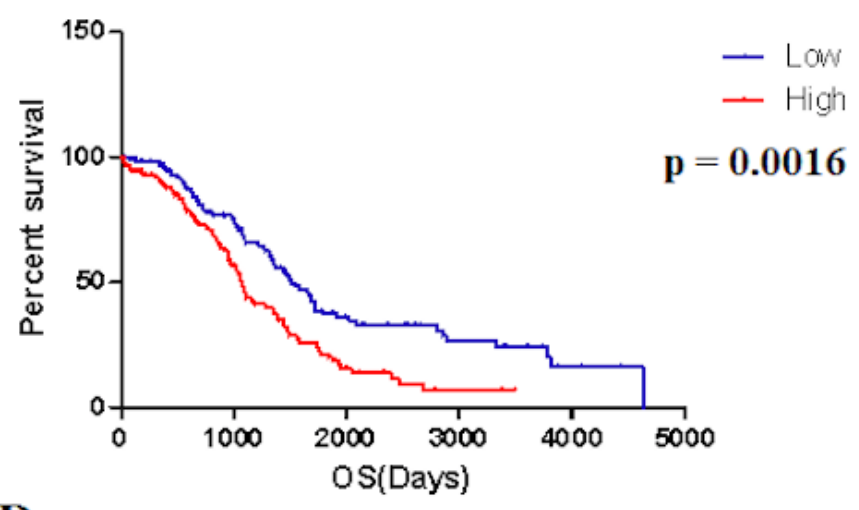

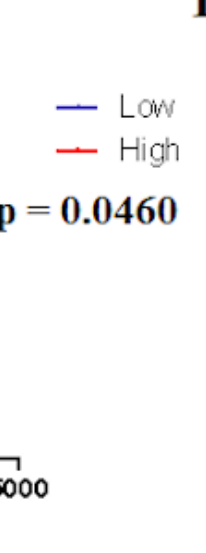

F

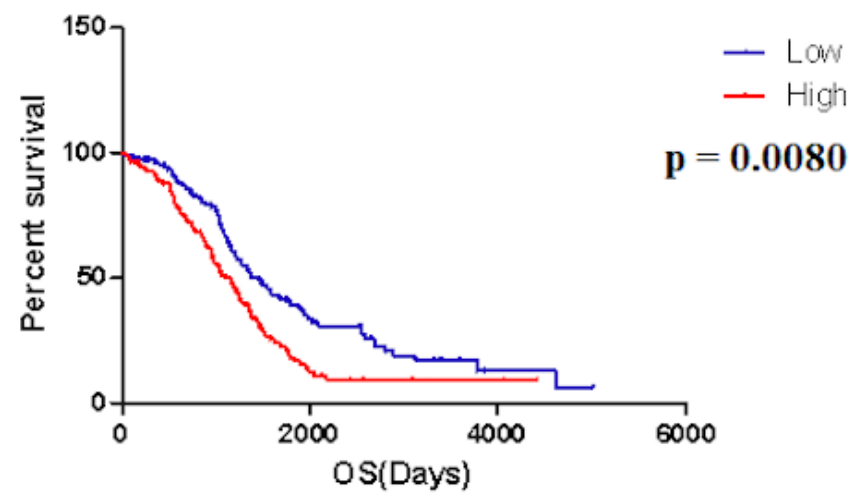

ZFP36

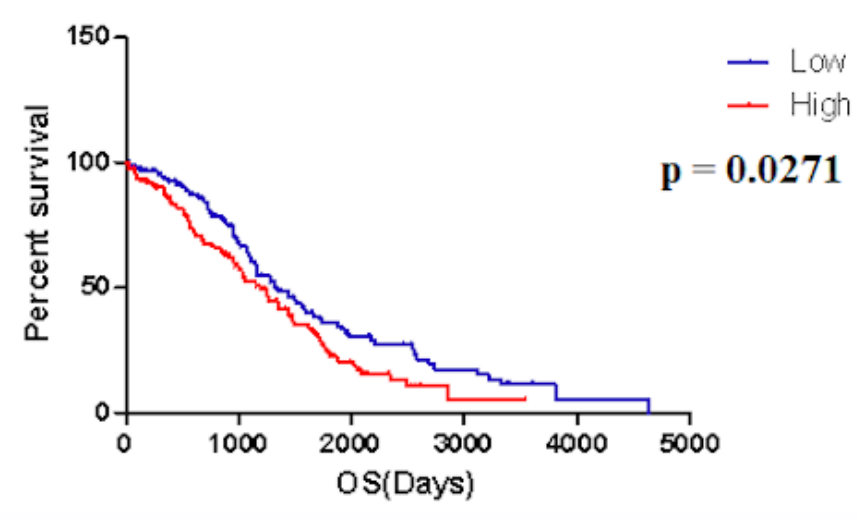

\section{Figure 4}

Correlation of expression of DEGs with OV prognosis in TCGA. Kaplan-Meier survival curves of the selected DEGs were ploted by the comparison of groups between low (blue line) and high (red line) gene expression. 


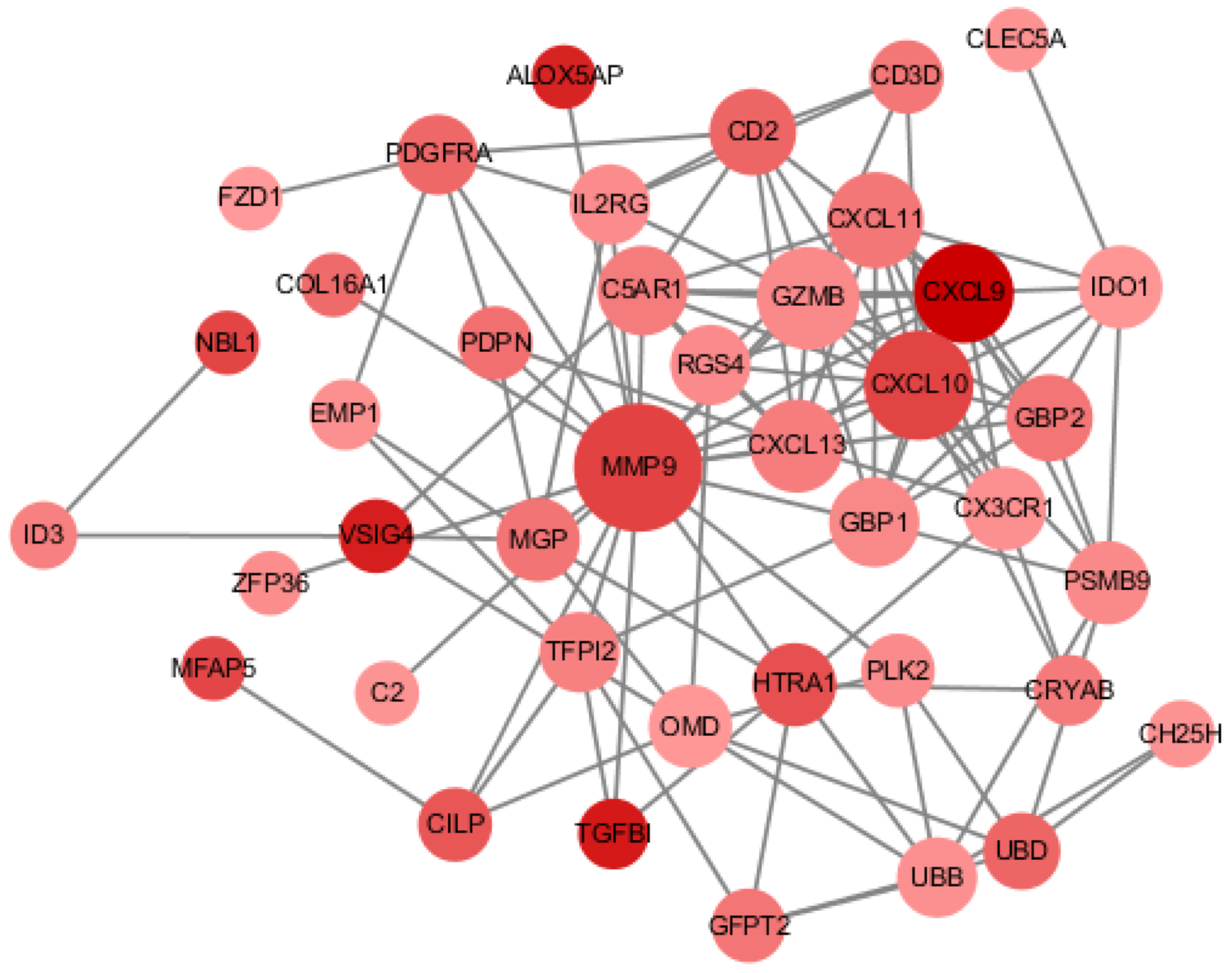

Figure 5

PPI networks of prognostic DEGs. The color of node was defined by log $(F C)$ value of prognostic DEGs and the size of node was based on degree value. 
A

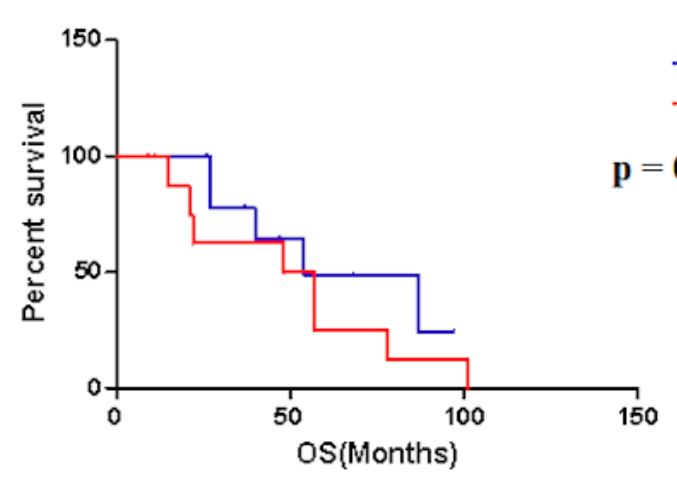

C

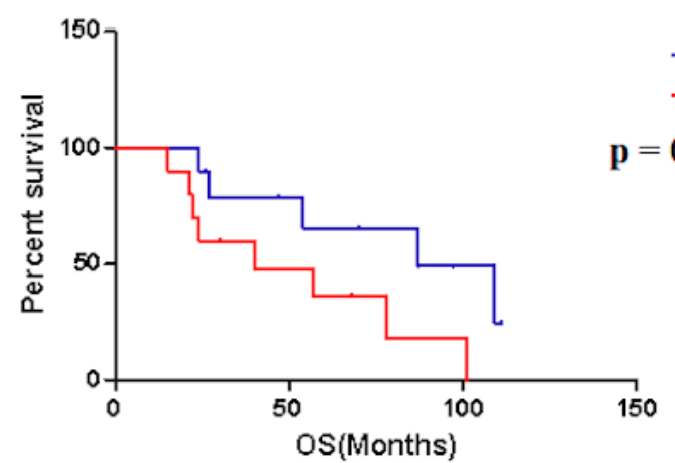

B

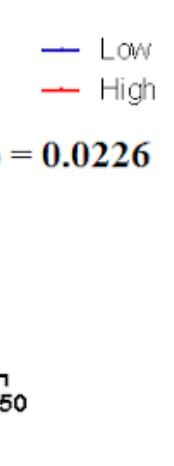

D

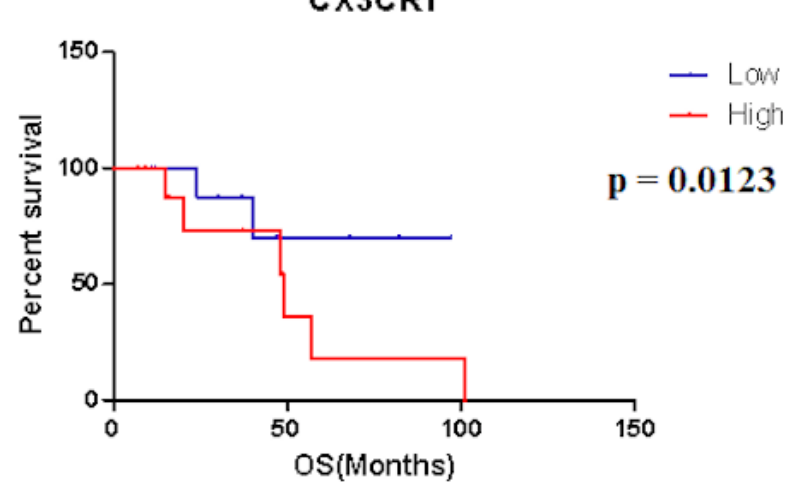

NBL1

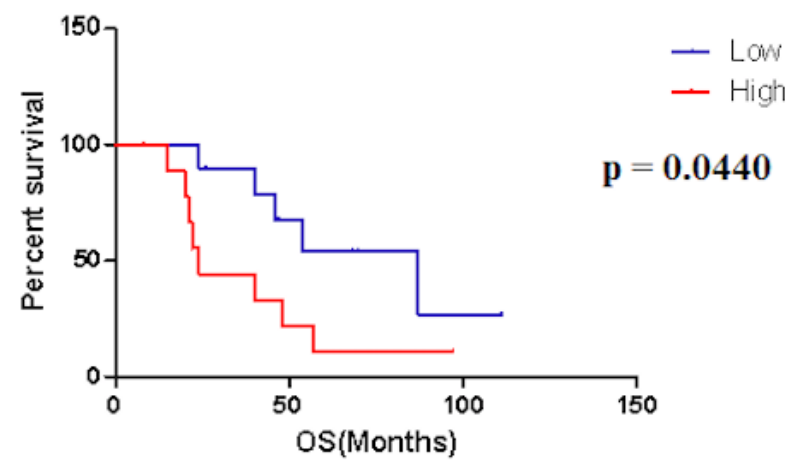

E

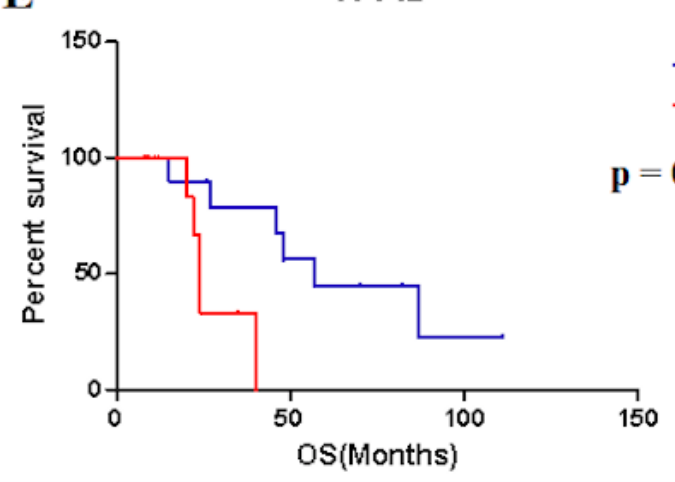

$\mathbf{F}$

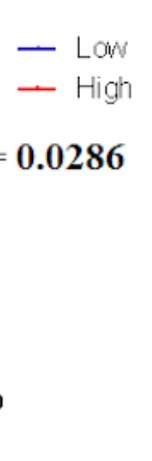

ZFP36

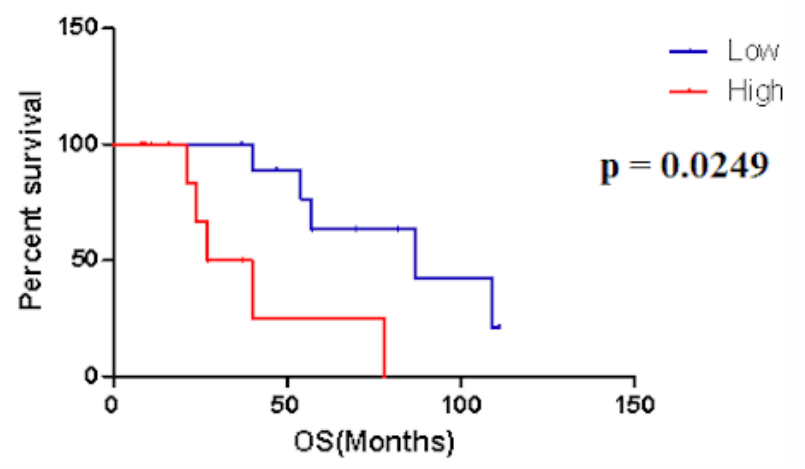

Figure 6

Correlation of expression of DEGs with OV prognosis from the GEO database. Kaplan-Meier survival curves of the selected DEGs were ploted by the comparison of groups between low (blue line) and high (red line) gene expression. 